\title{
Tunneling, dissipation, and superfluid transition in quantum Hall bilayers
}

\author{
Ziqiang Wang \\ Department of Physics, Boston College, Chestnut Hill, MA 02467
}

(Dated: November 13, 2018)

\begin{abstract}
We study bilayer quantum Hall systems at total Landau level filling factor $\nu=1$ in the presence of interlayer tunneling and coupling to a dissipative normal fluid. Describing the dynamics of the interlayer phase by an effective quantum dissipative $X Y$ model, we show that there exists a critical dissipation $\sigma_{c}$ set by the conductance of the normal fluid. For $\sigma>\sigma_{c}$, interlayer tunnel splitting drives the system to a $\nu=1$ quantum Hall state. For $\sigma<\sigma_{c}$, interlayer tunneling is irrelevant at low temperatures, the system exhibits a superfluid transition to a collective quantum Hall state supported by spontaneous interlayer phase coherence. The resulting phase structure and the behavior of the in-plane and tunneling currents are studied in connection to experiments.

PACS numbers: 73.43.Jn, 73.43.Nq, 73.21.-b, 64.60.Ak
\end{abstract}

A double-layer quantum Hall system at total Landau level filling factor $\nu=1$ exhibits a novel weak to strong coupling phase transition as a function of the interlayer separation $d / \ell$, where $\ell$ is the magnetic length. The weak-coupling (WC) phase at large $d / \ell$ corresponds to two weakly coupled compressible fluids with unquantized Hall conductance, whereas the strong-coupling (SC) phase at small $d / \ell$ is a $\nu=1$ quantum Hall liquid 1, 2, 3, 4]. If the layer index is regarded as a pseudospin index, the SC phase exhibits pseudospin ferromagnetism. Experimental evidence in support of spontaneous interlayer coherence in the SC phase was discovered recently. The interlayer tunneling conductance shows a sharp zerobias peak reminiscent of the DC Josephson effect [5], consistent with spontaneous breaking of the gauge symmetry associated with the charge difference between the layers [6, 7]. The fact that the zero-bias peak maintains a finite height and a nonzero width at low temperatures signals the importance of dissipation and generates a debate on whether there is true superfluidity in the presence of interlayer tunneling [12]. Considerable theoretical efforts have been devoted to understanding the effects of dissipation on the tunneling conductance $[8,9,10,11]$ and the nature of the weak to strong coupling transition 12, 13, 14, 15.

In this paper, we study the effects of interlayer tunneling on the (pseudospin) superfluid transition in the presence of dissipation in realistic samples. The source of dissipation we consider comes from the spatial fluctuations in the electron density near the transition [5]. As a consequence of such fluctuations, the width of the longitudinal drag resistance peak across a Hall drag transition is nonzero at low temperatures [16]. We thus view the bilayer near the SC to WC transition as an inhomogeneous mixture of SC droplets immersed in the background of the WC fluid. This picture was introduced recently by Stern and Halperin [17] to describe the enhancement of the interlayer Coulomb drag near the transition [16]. In that work, the transition and the associated transport properties were treated in terms of classical percolation of the SC droplets. Here, we study the effects of quantum fluctuations by exploring the analogy to granular superconductors near the superconductor-insulator transition 18, 19, 20, 21, 22, 23. Specifically, we consider a two-dimensional array of SC droplets embedded in the dissipative environment of the background diffusive normal fluid. The SC droplets are coupled by the Josephson coupling $J$, and to the $\mathrm{WC}$ dissipative normal fluid capacitively. After integrating out the WC electrons, we arrive at a dissipative, quantum $X Y$ model for the dynamics of the phases on the SC droplets. The interlayer tunneling $t$ plays the role of a symmetry breaking field which, in the absence of dissipation, is relevant in the renormalization group (RG) sense and destroys the putative superfluid transition. In the presence of dissipation, characterized by the pseudospin conductance $\sigma$ of the normal fluid, we find that quantum phase fluctuations lead to a critical dissipation $\sigma_{c}$. For $\sigma>\sigma_{c}, t$ is relevant and flows to large values at low temperatures. The system develops a mass gap associated with the symmetric-antisymmetric tunnel splitting of the single-particle band and is in the $\nu=1$ integer quantum Hall state. For $\sigma<\sigma_{c}$, the interlayer tunneling is irrelevant, $t$ scales to zero in the low temperature limit. We show that in this case the system undergoes a zero-temperature superfluid transition to a collective quantum Hall state with spontaneous interlayer phase coherence when $J$ exceeds a critical value $J_{c}$. The tunneling current is found to exhibit non-monotonic temperature dependence near the transition.

We begin with the action for a Josephson junction array of SC droplets (setting $\hbar=1$ ),

$$
\begin{aligned}
S_{s c}[\phi] & =\int_{0}^{\beta} d \tau\left[\frac{1}{2 U} \sum_{i}\left(\frac{\partial \phi_{i}}{\partial \tau}\right)^{2}+J \sum_{\langle i, j\rangle}\left(1-\cos \Delta \phi_{i j}\right)\right. \\
& \left.-t \sum_{i} \cos \phi_{i}(\tau)\right]
\end{aligned}
$$

where $\phi_{i}$ is the phase of the interlayer excitonic order parameter on the $i$-th droplet, $\left\langle\psi_{1}^{\dagger}\left(r_{i}\right) \psi_{2}\left(r_{i}\right)\right\rangle \propto e^{i \phi_{i}}$ with $\psi_{\alpha}^{\dagger}$ creating an electron in layer- $\alpha[3,4]$. The nearest 
neighbor droplets are coupled by Josephson coupling $J$ in Eq. (11), where $\Delta \phi_{i j}=\phi_{i}-\phi_{j}$, and $U=e^{2} / C_{s}$ is the charging energy set by the droplet capacitance $C_{s} \sim$ $\varepsilon \xi^{2} / d$ with $\xi$ the average droplet size. The last term in Eq. (11) represents the interlayer tunneling $t=N_{0} \Delta_{S A S}$ with $\Delta_{S A S}$ the symmetric-antisymmetric band splitting and $N_{0}$ the average number of electrons per droplet.

The SC droplets and the WC background fluid are not at the same potential. Denoting the phase of the WC electrons by $\varphi$ [24], there is a voltage drop $\left(\partial \phi_{i} / \partial \tau-\right.$ $\left.\partial \varphi_{i} / \partial \tau\right) / e$ across the 2D Thomas-Fermi screening length $\lambda_{T F}=1 / 4 \pi e^{2}(d n / d \mu)$ around the $i$-th droplet, where $d n / d \mu$ is the compressibility of the normal fluid. The action governing their capacitive coupling is thus

$$
S_{s c-w c}[\phi, \varphi]=\int_{0}^{\beta} d \tau \sum_{i} \frac{1}{2} \frac{C_{w}}{e^{2}}\left(\frac{\partial \phi_{i}}{\partial \tau}-\frac{\partial \varphi_{i}}{\partial \tau}\right)^{2} .
$$

where the effective capacitance $C_{w} \sim 1 / \lambda_{T F}$. The phase of the dissipative normal fluid is damped and follows the diffusive (Ohmic) dynamics,

$$
S_{w c}[\varphi]=\frac{1}{2 \beta} \sum_{n} \sum_{\langle i, j\rangle} \sigma\left|\omega_{n} \| \Delta \varphi_{i j}\left(\omega_{n}\right)\right|^{2},
$$

where $\omega_{n}=2 \pi n / \beta$ is the Matsubara frequency and $\sigma$ is the pseudospin conductivity in units of $e^{2} / \hbar$. The total action of the system is

$$
S=S_{s c}[\phi]+S_{s c-w c}[\phi, \varphi]+S_{w c}[\varphi] .
$$

To obtain an effective action for the phase of the SC droplets, we integrate out the phase $\varphi$ of the WC electrons in Eq. (4) using Eqs. (3) and (2). The result is

$$
\begin{aligned}
S_{\mathrm{eff}} & =\frac{1}{2 \beta} \sum_{q} \sum_{n} \Pi\left(q, \omega_{n}\right)\left|\phi\left(q, \omega_{n}\right)\right|^{2} \\
& +\int_{0}^{\beta} d \tau\left[J \sum_{\langle i, j\rangle}\left(1-\cos \Delta \phi_{i j}\right)-t \sum_{i} \cos \phi_{i}\right],
\end{aligned}
$$

where

$$
\Pi\left(q, \omega_{n}\right)=\frac{\omega_{n}^{2}}{U}+\frac{\sigma \Delta(q)}{\left|\omega_{n}\right|+D \Delta(q)} \omega_{n}^{2} .
$$

In Eq. (6), $D=e^{2} \sigma / C_{w}$ is the diffusion constant, and $\Delta(q)=4-2\left[\cos \left(q_{x} a\right)+\cos \left(q_{y} a\right)\right]$ with $a$ the average distance between the SC droplets, set to unity hereafter. Note that since dissipation implies that charges can be transferred continuously, the phase $\phi$ is noncompact 18 , 19], and the action (5) is in fact a dissipative quantum Sine-Gordon model in $2+1$ dimensions.

The phase fluctuations described by Eq. (6) have two very different dynamic regimes. (i) For fluctuations in the quantum regime, $\left|\omega_{n}\right|>D \Delta(q)$,

$$
\Pi\left(q, \omega_{n}\right)=\frac{\left|\omega_{n}\right|}{U}\left[\left|\omega_{n}\right|+\sigma U \Delta(q)\right], \quad\left|\omega_{n}\right|>D \Delta(q),
$$

the phase dynamics is dissipative. (ii) For $\left|\omega_{n}\right|<D \Delta(q)$, the level spacing is larger than the frequency and dissipation is inactive. The system returns to the charging regime with

$$
\Pi\left(q, \omega_{n}\right)=\left(C_{s}+C_{w}\right) \frac{\omega_{n}^{2}}{e^{2}} \equiv \frac{\omega_{n}^{2}}{U_{r}}, \quad\left|\omega_{n}\right|<D \Delta(q) .
$$

In this case, the WC normal fluid merely screens the electrostatic interaction of the exciton-pairs. The dynamics of the phase is again capacitive, with a renormalized charging energy $U_{r}$ determined by putting the capacitors in parallel. As a result, the phase space for the damping of the SC phase is limited to the quantum fluctuation regime, in contrast to resistive shunted superconducting grains [18, 19]. Writing $\phi\left(q, \omega_{n}\right)=h\left(q,\left|\omega_{n}\right|>\right.$ $\left.D q^{2}\right)+\theta\left(q,\left|\omega_{n}\right|<D q^{2}\right)$, our strategy now is to integrate out the dissipative fluctuations $h$ in regime (i) to arrive at an effective theory of capacitive junctions for $\theta$ in regime (ii) with renormalized parameters. The correlation function $G_{h}\left(\mathbf{r}_{\mathbf{i}}-\mathbf{r}_{\mathbf{j}}, \tau-\tau^{\prime}\right)=\left\langle h_{i}(\tau) h_{j}\left(\tau^{\prime}\right)\right\rangle$ is given by

$$
G_{h}(\mathbf{r}, \tau)=\frac{2}{\beta} \sum_{n \geq 0} \frac{U}{\omega_{n}} \sum_{q<\Lambda_{\omega}} \frac{\cos \left(\omega_{n} \tau\right) e^{i \mathbf{q} \cdot \mathbf{r}}}{\omega_{n}+\sigma U \Delta(q)} .
$$

Here the cutoff $\Lambda_{\omega}$ for the momentum summation is specified by the condition $D \Delta\left(\Lambda_{\omega}\right)=\left|\omega_{n}\right|$ in Eq. (7).

Consider first the renormalization of the tunneling term in Eq. (5). Within the standard Gaussian approximation, $t\left\langle\cos \phi_{i}\right\rangle_{h}=t\left\langle\cos \left(h_{i}+\theta_{i}\right)\right\rangle_{h} \equiv t_{r} \cos \theta_{i}$. The renormalized tunneling is given by $t_{r}=t \exp \left(-W_{t}\right)$, where the "Debye-Waller" factor

$$
W_{t}=\frac{1}{2}\left\langle h_{i}^{2}(\tau)\right\rangle=\frac{1}{2} G_{h}(0,0),
$$

represents the renormalization of the tunneling due to dissipative phase fluctuations. Evaluating $G_{h}(0,0)$ using Eq. (9) in the continuum limit shows that $W_{t}$ is logarithmically divergent at low temperatures,

$$
W_{t}=-\frac{1}{8 \pi^{2} \sigma} \ln \left(1+C_{w} / C_{s}\right) \ln \left(T \tau_{0}\right),
$$

where $\tau_{0}$ is a microscopic, short time scale serving as a high frequency cutoff. This singularity is a form of the orthogonality catastrophe: the fast voltage fluctuations created by the coherent tunneling of the electrons between the layers cannot be followed adiabatically by the dissipative dynamics. As a result, the renormalized tunneling vanishes according to a power law,

$$
t_{r}=t\left(T \tau_{0}\right)^{2 \sigma_{c} / \sigma},
$$

at low temperatures, where $\sigma_{c}=\left(1 / 16 \pi^{2}\right) \ln \left(1+C_{w} / C_{s}\right)$. The renormalization of $J$ can be obtained similarly by writing $J\left\langle 1-\cos \Delta \phi_{i j}\right\rangle_{h}=J_{r}\left(1-\cos \Delta \theta_{i j}\right)$, with $J_{r}=$ $J \exp \left(-W_{J}\right)$. However, the limited phase space for the dissipative fluctuations renders

$$
W_{J}=\frac{1}{8 \pi^{2}} \int_{0}^{1 / \tau_{0}} \frac{U}{\omega} \int_{0}^{\sqrt{\omega / D}} q d q \frac{q^{2}}{\omega+\sigma U q^{2}},
$$


free of the infrared singularity and $W_{J}=\left(1 / 16 \pi^{2}\right)[1-$ $\left.\left(C_{s} / C_{w}\right) \ln \left(1+C_{w} / C_{s}\right)\right]\left(1 / D \tau_{0}\right)$. This is in sharp contrast to resistive shunted Josephson junctions where the unlimited dissipative fluctuations lead to logarithmic corrections to the Josephson coupling [18, 19].

Eq. (12) suggests that the scaling dimension of the tunneling $t$ is $-2 z_{T} \sigma_{c} / \sigma$, where $z_{T}=2$ is the thermal exponent relating the temperature scale to the length scale. Taking into account its naive dimension, we obtain the RG dimension for $t, D_{t}=2+z_{T}-2 z_{T} \sigma_{c} / \sigma=4\left(1-\sigma_{c} / \sigma\right)$. Thus, $\sigma_{c}$ is identified with the critical dissipation that separates a weak-dissipation phase with irrelevant interlayer tunneling for $\sigma<\sigma_{c}$ from a strong-dissipation phase for $\sigma>\sigma_{c}$ where the interlayer tunneling is relevant. This conclusion can be made more explicit if the DebyeWaller factor $W_{t}$ in Eq. (10) is evaluated self-consistently by including the mass term $\sqrt{U t_{r}}$ in the $h$-field propagator in Eq. (9), which replaces $T$ as the cutoff for the logarithmic divergence in Eq. (11) at low temperatures. We then obtain $t_{r}=t\left(U t_{r} \tau_{0}^{2}\right)^{\sigma_{c} / \sigma}$ at $T=0$ in place of Eq. (12). This equation has a physical, nonzero solution, $t_{r}=\left(1 / \tau_{0}\right)\left(U \tau_{0}\right)^{\sigma_{c} /\left(\sigma-\sigma_{c}\right)}\left(t \tau_{0}\right)^{\sigma /\left(\sigma-\sigma_{c}\right)}$ only for $\sigma>\sigma_{c}$, whereas $t_{r}=0$ for $\sigma<\sigma_{c}$, consistent with the conclusion drawn from the RG dimension. The critical dissipation depends on the ratio $C_{w} / C_{s}$ and is not universal.

The renormalized action for the phase $\theta_{i}$ in the charging regime has the same form as Eq. (10), but with renormalized parameters $U_{r}, J_{r}$, and $t_{r}$. We now study the development of interlayer phase coherence characterized by a nonzero order parameter $\left\langle\cos \theta_{i}\right\rangle$. It turns out that the interlayer coherence can be driven by either the interlayer tunneling or the Josephson coupling between the droplets, depending on the degree of dissipation. To illustrate this, we set $J=0$ which gives the Lagrangian $\mathcal{L}=\left(1 / 2 U_{r}\right)\left(\partial \theta_{i} / \partial \tau\right)^{2}-t_{r} \cos \theta_{i}$. The system thus turns into decoupled droplets, or single vertical junctions, and the interlayer tunneling $t_{r}$ serves as the Josephson coupling. The competition between the charging energy and the Josephson coupling of a single junction in granular metals was studied by Abeles [20]. For the phase coherence to develop at $T=0$, the frequency of the quantum zero point oscillations, $\omega_{p}=\sqrt{t_{r} U_{r}}$ (interlayer Josephson plasma frequency) must not exceed the Josephson coupling strength $t_{r}$, i.e. $t_{r}>U_{r}$. This condition is satisfied for strong dissipation since the renormalized $t_{r}$ scales to large values at low temperatures. Thus for $\sigma>\sigma_{c}$, the interlayer tunneling drives the layers phase coherent even in the absence of coupling between the pseudospins in the $2 \mathrm{D}$ plane. The system is in an integer quantum Hall state due to the relevant symmetric-antisymmetric tunneling splitting of the single-particle band in this regime.

We now focus on the case of weak dissipation, $\sigma<\sigma_{c}$, where the renormalized tunneling $t_{r}$ scales to zero according to Eq. (12). In this case, the Abeles criterion cannot be satisfied at low temperatures and interlayer tunneling does not lead to phase coherence. However, we show that a zero temperature pseudospin superfluid transition can occur through Josephson coupling between the droplets. Following Doniach 21], Efetov 22], and Simánek [23], we replace $J_{r} \cos \left(\theta_{i}-\theta_{j}\right) \rightarrow J_{r}\left\langle\cos \theta_{i}\right\rangle \cos \theta_{j}$ in Eq. (1). The array is thus described by an effective single-droplet theory specified by the self-consistency equation for the superfluid order parameter $\Psi=2\left\langle\cos \theta_{i}\right\rangle$,

$$
\begin{aligned}
\Psi & =\frac{2}{Z_{0}} \int \mathcal{D}[\theta] \cos \theta_{j} e^{-\int_{0}^{\beta} d \tau \sum_{i} L_{0}\left[\theta_{i}(\tau)\right]} \\
L_{0} & =\frac{1}{2 U_{r}}\left(\frac{\partial \theta_{i}}{\partial \tau}\right)^{2}+2 \Psi J_{r} \cos \theta_{i}-t_{r} \cos \theta_{i},
\end{aligned}
$$

where $Z_{0}$ is the meanfield partition function. Since the renormalized tunneling $t_{r}$ is small at low temperatures, $\Psi$ can be obtained to leading order in $t_{r}=t\left(T \tau_{0}\right)^{\mu}, \mu=$ $2 \sigma_{c} / \sigma>2$ by linearizing Eq. (14),

$$
\Psi=t\left(T \tau_{0}\right)^{\mu} \chi(T), \quad \chi(T)=\frac{\chi_{0}(T)}{1-2 J_{r} \chi_{0}(T)} .
$$

Here $\chi(T)$ is the RPA-like susceptibility and

$$
\chi_{0}(T)=2 \int_{0}^{\beta} d \tau\langle\cos \theta(\tau) \cos \theta(0)\rangle .
$$

Evaluating $\chi_{0}$ using the Gaussian fluctuation in Eq. (15), $\langle\phi(\tau) \phi(0)\rangle=\left(U_{r} / \beta\right) \sum_{n} \cos \left(\omega_{n} \tau\right) / \omega_{n}^{2}$, We obtain

$$
\chi_{0}(x)=\int_{0}^{\beta} d \tau e^{-\frac{U_{r}}{2 \beta} \tau(\beta-\tau)}=\frac{8}{U_{r}} y(x), \quad x=\sqrt{\frac{U_{r}}{8 T}},
$$

where $y(x)=x e^{-x^{2}} \int_{0}^{x} d z e^{z^{2}}$, a hypergeometric function, has the limiting behavior $\lim _{x \rightarrow \infty} y(x)=1 / 2$. At $T=0$, the onset of spontaneous interlayer phase coherence is determined by $2 J_{r} \chi_{0}(\infty)=1$, leading to a quantum critical point (QCP) at $J_{r}^{c}=U_{r} / 8$, which separates a SC phase with spontaneous interlayer phase coherence for $J_{r}>J_{r}^{c}$ from an interlayer incoherent WC phase for $J_{r}<J_{r}^{c}$. Since $U \sim\left(e^{2} / \varepsilon \ell\right)(\ell / \xi)^{2}(d / \ell)$ and $J \sim e^{2} / \varepsilon \ell$, this condition corresponds to the existence of a critical layer separation $d_{c} / \ell$ below which $2 \mathrm{D}$ superfluidity develops, in qualitative agreement with experiments [2, [5]. In the present theory based on the droplet picture, the critical layer separation is not universal, but depends on the average size of the droplets in the critical regime, a prediction that can be verified by experiments.

At finite temperatures, since the symmetry-breaking field $t_{r}$ is finite, one does not expect phase transitions in the usual sense. However, the divergence of the susceptibility $\chi$ at finite temperatures when $1=2 J_{r} \chi_{0}\left(T_{c}\right)$ would signal the onset of rapid growth of the magnetization $\Psi$ associated with a crossover or a metamagnetic-like transition. The effects of a finite but small $t_{r}$ on $T_{c}$ near the QCP can be estimated from the nonlinear susceptibility by including the contributions from interlayer tunneling 
$t_{r}$ in $\chi_{0}(T)$. To leading order in $t$ and at low temperatures, we obtain

$$
T_{c} \simeq \frac{1}{\tau_{0}}\left[\left(\frac{3}{2 t \tau_{0}}\right)\left(\frac{J_{r}^{c}-J_{r}}{J_{r}}\right)\right]^{1 /(\mu-1)}, \quad J_{r}<J_{r}^{c} .
$$

Although the tunneling is irrelevant in the RG sense, it suppresses thermal phase fluctuations and moves the phase boundary from $J_{r}^{c}$ at $T=0$ to smaller values of $J_{r}$ at finite temperatures. Thus, for $\sigma<\sigma_{c}$ and $J_{r}$ less than but close to the QCP, the system exhibits an interesting reentrant behavior (incoherent - coherent - incoherent) as the temperature is lowered.

The in-plane and the interlayer tunneling currents behave qualitatively differently in the interlayer coherent phase. The pseudospin current is given by the interdroplet current $J_{r}\left\langle\sin \left(\theta_{i}-\theta_{j}\right)\right\rangle$ times the average number of droplets normal to the direction of the current flow. Separating $\theta$ into a fluctuating part $\tilde{\theta}$ and a slowly varying classical part $\theta_{0}$, and average over $\tilde{\theta}$ within the above meanfield theory, we obtain $I_{2 d}=2 \sqrt{N_{d}} J_{r} \Psi^{2}\left(d \theta_{0} / d x\right)$ in the continuum limit, where $N_{d}$ is the total number of droplets. Thus the in-plane critical current increases monotonically with $\Psi$ as the temperature is lowered. The superfluid stiffness can be identified as $\rho_{s}=J_{r} \Psi^{2}$. The theory predicts that the tunneling current, on the other hand, exhibits a non-monotonic temperature dependence. In an ideal setup for tunneling, the current flows in the interlayer direction and is given by $I_{T}=N_{d} t_{r}\langle\sin \theta\rangle=I_{c} \sin \theta_{0}$, where the $I_{c}=N_{d} t\left(T \tau_{0}\right)^{\mu} \Psi$. The tunneling current thus decreases initially with decreasing temperatures near the transition. However, at low temperatures or well inside the SC phase, the inplane superfluid stiffness $\rho_{s}$ will cut off the logarithmic singularity in the dissipation induced Debye-Waller factor for tunneling, $W_{t}$ in Eq. (11), such that $T$ is replace by $\rho_{s}$ in Eq. (12). This leads to $I_{c}=N_{d} t\left(J_{r} \Psi^{2} \tau_{0}\right)^{\mu} \Psi$ which then grows with the superfluid order parameter upon further lowering of the temperature.

In conclusion, the interplay between interlayer tunneling and quantum dissipation is studied in quantum Hall bilayers at $\nu=1$. We have shown the existence of a critical dissipation separating a strong dissipation phase I from a weak dissipation phase II. Phase I is an integer quantum Hall state driven by interlayer tunneling and the development of a single-particle gap. In phase II, the tunneling is renormalized to zero at low temperatures by quantum fluctuations. This enables a quantum phase transition between a compressible WC phase II(a) and a SC collective quantum Hall state II(b) supported by spontaneous interlayer phase coherence. The observation of the linearly dispersing Goldstone mode with inplane magnetic field [5] is consistent with the superfluid phase II(b). However, the fact that a weaker conductance peak remains at zero-bias suggests that in-plane superfluidity is perhaps not the only driving force behind interlayer coherence and the system might not be far from phase I where interlayer tunneling itself is able to produce coherent zero-bias tunneling. Future experiments on the temperature dependence of the residual conductance peak may help resolve its origin. We have not addressed the finite height and the nonzero width of the zero-bias conductance peak. The lack of an apparent DC Josephson effect may be accounted for by the absence of a finite temperature Kosterlitz-Thouless transition due to nonzero interlayer tunneling and/or the presence of this and other forms of dissipation, such as the vortices induced by density inhomogeneities 8, , 9, 10, 11, 12] not considered in the present theory.

The author thanks L. Balents, J. Eisenstein, S. M. Girvin, and D. N Sheng for discussions. This work is supported in part by DOE grant No. DE-FG02-99ER45747 and ACS grant No. 39498-AC5M.

[1] J. P.Eisenstein et. al., Phys. Rev. Lett. 68, 1383 (1992).

[2] S. Q. Murphy et al., Phys. Rev. Lett. 72, 728 (1994);

[3] For reviews, see S. M. Girvin and A. H. MacDonald, in Perspectives in Quantum Hall Effect, edited by S. Das Sarma and A. Pinczuk (Wiley, New York, 1997).

[4] K. Moon et al., Phys. Rev. B 51, 5138 (1995); K. Yang et al., ibid. 54, 11644 (1996).

[5] I. B. Spielman et al., Phys. Rev. Lett. 84, 5808 (2000); 87, 036803 (2001).

[6] H. Fertig, Phys. Rev. B40, 1087 (1989).

[7] X. G. Wen and A. Zee, Phys. Rev. Lett. 69, 1811 (1992); Phys. Rev. B47, 2265 (1993); Z. F. Ezawa and A. Iwazaki, Phys. Rev. B48, 15189 (1993); 47, 7295 (1992).

[8] L. Balents and L. Radzihovsky, Phys. Rev. Lett. 86, 1825 (2001).

[9] A. Stern et al., Phys. Rev. Lett. 86, 1829 (2001).

[10] M. Fogler and F. Wilczek, Phys. Rev. Lett. 86, 1833 (2001).

[11] H. A. Fertig and J. P. Straley, Phys. Rev. Lett. 91 046806, (2003).

[12] Y. N. Joglekar and A. H. MacDonald, Phys. Rev. B64, 155315 (2001); Phys. Rev. Lett. 87, 196802 (2001).

[13] J. Schliemann, S. M. Girvin, and A. H. MacDonald, Phys. Rev. Lett. 86, 1849 (2001).

[14] N. E. Bonesteel et. al., Phys. Rev. Lett. 77, 3009 (1996); Y. B. Kim et al., Phys. Rev. B63, 205315 (2001); M. Veillette, L. Balents and M. P. A. Fisher, ibid. 66, 155401 (2002). D. W. Wang, E. Demler, and S. Das Sarma, cond-mat/0303324 J. Ye, cond-mat/0302558

[15] D. N. Sheng, L. Balents, and Z. Wang, Phys. Rev. Lett. 91, 116802 (2003).

[16] M. Kellogg et. al., Phys. Rev. Lett. 90, 246801 (2003).

[17] A. Stern and B. I. Halperin, Phys. Rev. Lett. 88, 106801 (2002).

[18] S. Chakravarty et. al., Phys. Rev. Lett. 56, 2303 (1986).

[19] M. P. A. Fisher, Phys. Rev. B36, 1917 (1987).

[20] B. Abeles, Phys. Rev. B15, 2828 (1977).

[21] S. Doniach, Phys. Rev. B24, 5063 (1981).

[22] K. B. Efetov, Sov. Phys. JETP 51, 1015 (1980).

[23] E. Ŝimánek, Phys. Rev. B32, 500 (1985).

[24] U. Eckern, G. Schön, and V. Ambegaokar, Phys. Rev. 
B30, 6419 (1984). 